Provided for non-commercial research and education use. Not for reproduction, distribution or commercial use.

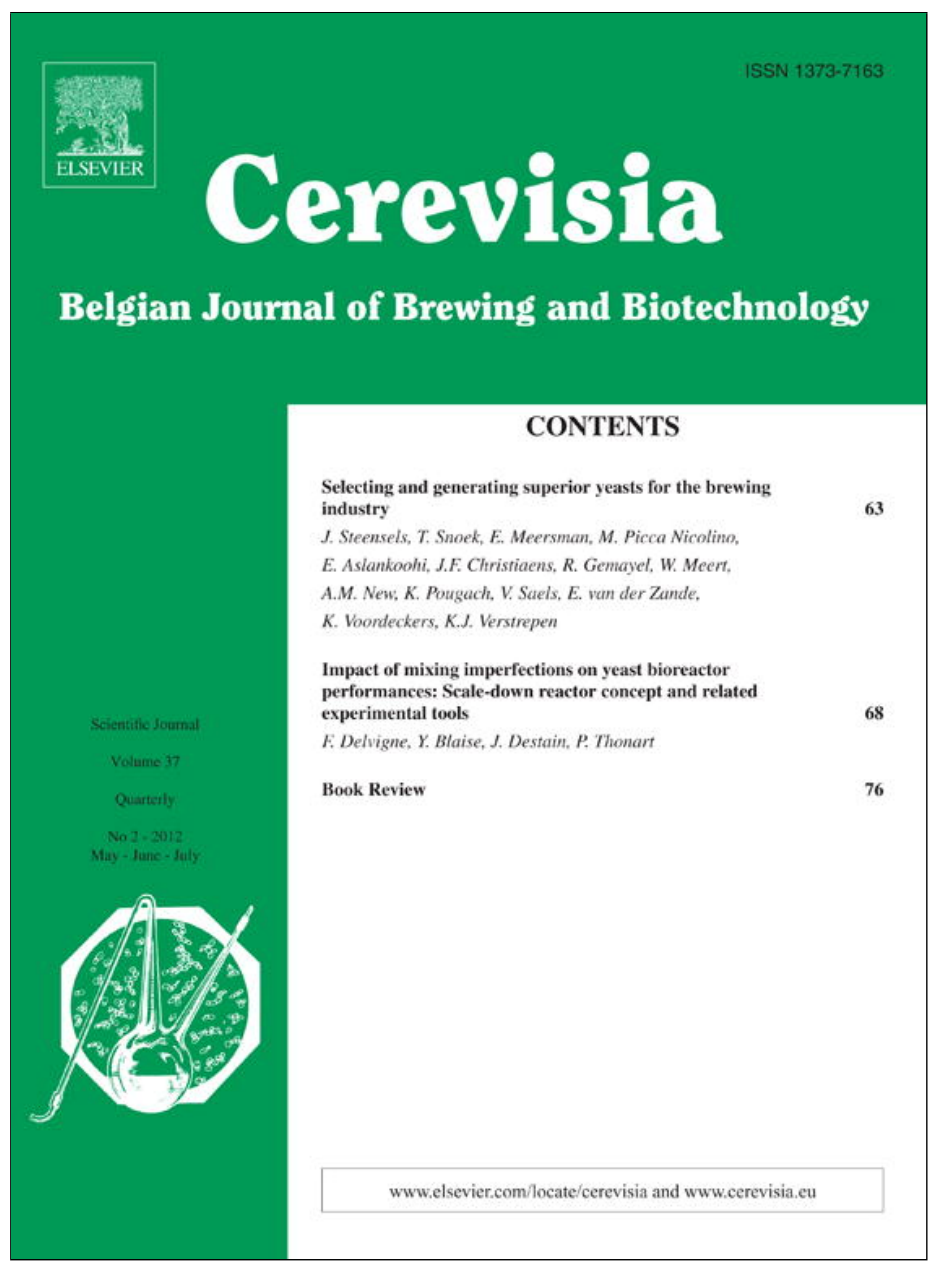

This article appeared in a journal published by Elsevier. The attached copy is furnished to the author for internal non-commercial research and education use, including for instruction at the authors institution and sharing with colleagues.

Other uses, including reproduction and distribution, or selling or licensing copies, or posting to personal, institutional or third party websites are prohibited.

In most cases authors are permitted to post their version of the article (e.g. in Word or Tex form) to their personal website or institutional repository. Authors requiring further information regarding Elsevier's archiving and manuscript policies are encouraged to visit:

http://www.elsevier.com/copyright 


\title{
Impact of mixing imperfections on yeast bioreactor performances: Scale-down reactor concept and related experimental tools
}

\author{
Frank Delvigne*, Yannick Blaise, Jacqueline Destain, Philippe Thonart \\ Université de Liège, Gembloux Agro-Bio Tech, Unité de Bio-industries, Passage des déportés, 2, B-5030, Belgium
}

\section{A R T I C L E I N F O}

\section{Keywords:}

Markov

Dissolved oxygen control

Environmental state

Scale-down

\begin{abstract}
A B S T R A C T
A method combining environmental data extracted from the dissolved oxygen profile of a fed-batch bioreactor and a dynamic discrete Markov chain model has been presented in order to give more insight about the glucose and dissolved oxygen fluctuations experienced by the microorganisms during cultivation in heterogeneous bioreactor. The fed-batch cultivation of Saccharomyces cerevisiae has been performed in a well-mixed and a partitioned scale-down reactor (SDR). The analysis of the environmental sequences has shown extended time lengths for the glucose availability and depletion sequences in the case of the SDR under a DO-controlled fed-batch culture. The Markov chain model developed in this work is able to capture the stochastic environmental events, i.e. in our case the environmental states experienced by the microorganisms crossing the tubular part of the SDR. The simulation results show clearly an extension of the starvation periods in the case of the culture performed in the SDR. The simulations have been performed at the single cells level allowing future improvements of our model and notably in the context of the population segregation phenomena occurring in fed-batch cultures. As a perspective, flow cytometry has been presented as a high-throughput analytical tool for the investigation of yeast physiology at the single cell level and in process-related conditions.
\end{abstract}

(C) 2012 the Associations of the Former Students of the Belgian Brewing Schools. Published by Elsevier B.V. All rights reserved.

\section{Introduction}

Saccharomyces cerevisiae is widely used at the industrial level for the production of alcohol, bulk biomass and more recently as a recombinant microorganism for the production of several foreign proteins (Junker, 2004). The metabolic characteristics of the strains impose a strict control of the environmental conditions in the bulk of the bioreactor. The bioreactor is generally recognized as a system exhibiting optimal hydrodynamic conditions in order to promote homogenous extracellular environment during the whole course of the culture. However, in practice, the extracellular conditions can be strongly heterogeneous leading to microbial stresses. These stresses induce different kind of modification at different physiological levels: metabolic shift, alteration of the genomic expression profile and population segregation (Gasch et al., 2000; Geladé et al., 2003; Henson et al., 2002; Müller and Lösche, 2004). These heterogeneities comes from several intrinsic, extrinsic and external sources, i.e. from the physiological processes themselves leading to the consumption of substrates and the excretion of metabolites to the physical processes involving the control

\footnotetext{
* Corresponding author. Tel.: +32 816223 11; fax: +32 81614222

E-mail address: F.Delvigne@ulg.ac.be (F. Delvigne).
}

systems and the reactor hydraulic behavior (Müller et al., 2010; Patnaik, 2006). This work will be mainly focused on the characterization of primary sources of noise, i.e. the external noise directly in relation with the homogenization performance of the bioreactor. A dissolved-oxygen controlled fed-batch culture of $S$. cerevisiae will be investigated. The DO-control is effective in order to optimize microbial growth and avoid secondary metabolism such as glucose overflow and ethanol formation during aerobic process (Cannizzaro and von Stockar, 2004). In addition, this regulation allow to track the dissolved oxygen and glucose profile experienced by the microbial cells during the culture and constitute thus a good model culture for the investigation of the interactions between the microbial cells and the environmental conditions generated by the bioreactor hydrodynamics. The methodology presented in this work is based on the mathematical manipulation of a set of data about the glucose availability from the DO profile. This methodology, combined with a stochastic model leads to the characterization of the exposure of individual microbial cells to concentration gradients and can be used in order to make the comparison between the environmental events (in relation with the dissolved oxygen and the glucose availability) occurring in a well-mixed reactor and in a two-compartment scale-down reactor (SDR) configured to generate flow imperfections. 


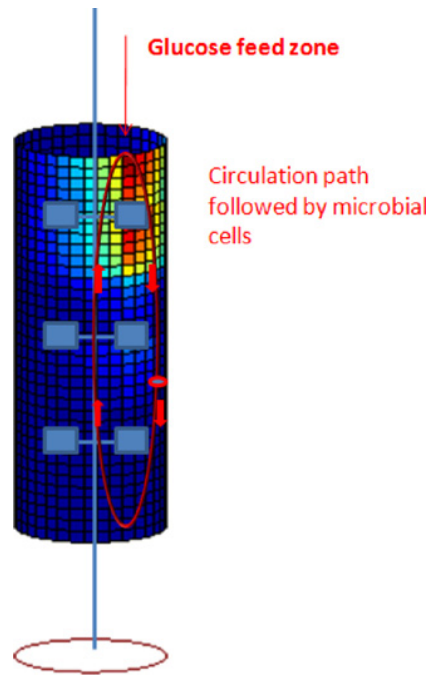

Fig. 1. Illustration of the exposure of microbial cells to glucose gradient concentration inside an industrial bioreactor operating in fed-batch mode. The color intensity is proportional to the glucose concentration and the figure shows that glucose accumulates at the level of the upper part of the bioreactor considering the lack at the level of the mixing efficiency of the system. (For interpretation of the references to color in this figure legend, the reader is referred to the web version of the article.)

\section{The scale-down reactor concept}

The main problem associated with bioprocesses scaling-up is the formation of concentration gradients inside large-scale bioreactors. These gradients induce various stresses at the level of microbial cells, such as glucose excess, glucose starvation, oxygen limitation, $\mathrm{pH}$ shock,... leading to deviation from the desired metabolism and in extreme case to a complete modification of the metabolism subsequent to a modification of the gene expression pattern. Studies have been mainly focused on glucose gradient appearing during fed-batch process (Delvigne et al., 2006), although other kinds of gradient have also been considered. The characterization of the exposure of microorganisms to gradients stress is not a trivial task, since several phenomena, including bioreactor mixing efficiency and microorganism circulation, are involved (Fig. 1). Scale-down reactors (SDR) have been used as an experimental predictive tool for the design of yeast-based bioprocesses (Lara et al., 2006). A SDR is a small scale bioreactor able to reproduce mixing imperfections encountered in large-scale bioreactors. One of the most used SDR design is presented in Fig. 2. This SDR

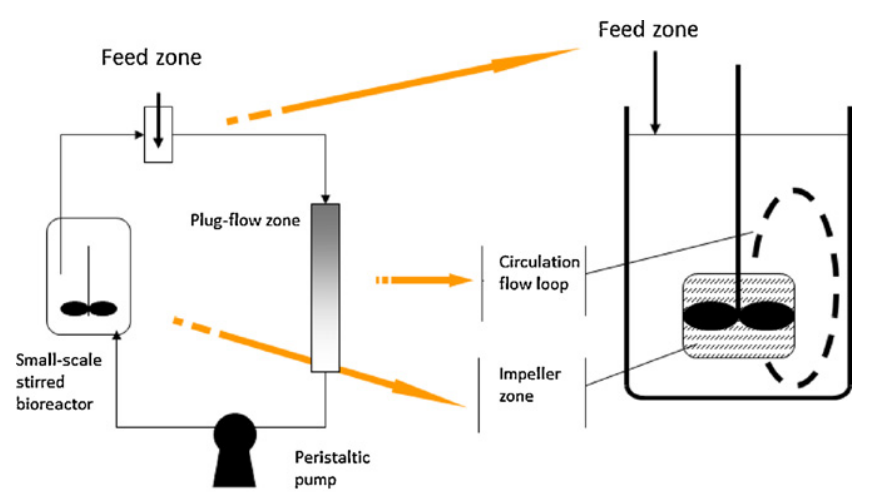

Scale-down reactor

Industrial

bioreactor

Fig. 2. Illustration of the scale-down reactor concept. A two-compartment reactor is able to reproduce the passage of a microorganism in different zone in a process analog to that encountered at the industrial scale. is composed of two compartments, i.e. a small-scale stirred bioreactor connected to a tubular plug-flow section. Controlled gradient can be generated at the level of the tubular recycle loop. By this way, the passage of microbial cells form one compartment to another can be experimentally reproduced and the corresponding physiological stresses can be investigated. However, the stochastic process by which microorganisms switch form a compartment to another is still difficult to represent. The main part of this paper deals with the design of a mathematical model able to simulate the passage of yeast cells from a compartment to another and the characterization of the substrate concentration profile encountered at the single-cell level.

\section{Materials and methods}

\section{Strain and bioreactor operating conditions}

S. cerevisiae (MUCL 43340), stored at $-80^{\circ} \mathrm{C}$ before use. The scale-down reactor was composed of a 201 stirred bioreactors (Biolafitte-France) with two turbines TD4-TD4. The regulation of temperature $\left(30^{\circ} \mathrm{C}\right), \mathrm{pH}(5.5)$ and dissolved oxygen was ensured by using a direct control system (ABB). Dissolved oxygen level was maintained above $30 \%$ saturation by the stirrer speed. Aeration was fixed at $1 \mathrm{vvm}$. The stirred reactor was connected by a peristaltic pump (Watson Marlow 325D) to a silicon pipe (internal diameter $=8 \mathrm{~mm}$, external diameter $=12 \mathrm{~mm}$, length $=7.5 \mathrm{~m}$, capacity $=0.377 \mathrm{l}$ ). The medium used was a CFM mineral medium, composed of (all compounds are expressed in $\left.\mathrm{gl}^{-1}\right): \mathrm{KH}_{2} \mathrm{PO}_{4}, 6.0 ;\left(\mathrm{NH}_{4}\right)_{2} \mathrm{SO}_{4}, 12.0 ; \mathrm{MgSO}_{4}$, 1.0; EDTA, 0.015; $\mathrm{ZnSO}_{4} \times 7 \mathrm{H}_{2} \mathrm{O}, 0.0045 ; \mathrm{MnCl}_{2} \times 4 \mathrm{H}_{2} \mathrm{O}, 0.001$; $\mathrm{CoCl}_{2} \times 6 \mathrm{H}_{2} \mathrm{O}, 0.0003 ; \mathrm{CuSO}_{4} \times 5 \mathrm{H}_{2} \mathrm{O}, 0.0003 ; \mathrm{Na}_{2} \mathrm{MoSO}_{4} \times 2 \mathrm{H}_{2} \mathrm{O}$, 0.004; $\mathrm{CaCl}_{2} \times 2 \mathrm{H}_{2} \mathrm{O}, 0.0045 ; \mathrm{FeSO}_{4} \times 7 \mathrm{H}_{2} \mathrm{O}, 0.003 ; \mathrm{H}_{3} \mathrm{BO}_{3}, 0.001$; D-biotin, 0.1; D,L-pantothenic acid, 0.001; nicotinic acid, 0.001; myo-inositol, 0.0025 ; thiamin, 0.001 ; pyridoxin, 0.001 ; paraaminobenzoic acid, 0.0002 . Glucose and yeast extract were added at final concentration of $5 \mathrm{gl}^{-1}$ each. The fed-batch solution was composed of (all compounds are expressed in $\left.\mathrm{gl}^{-1}\right)$ : $\left(\mathrm{NH}_{4}\right)_{2} \mathrm{SO}_{4}, 10.0$; $\mathrm{KH}_{2} \mathrm{PO}_{4}, 5.0 ; \mathrm{MgSO}_{4}, 0.9$; yeast extract, 5.0. Glucose was added at final concentration of $30 \mathrm{gl}^{-1}$.

\section{Characterization of the environmental sequences on the basis of the DO-profile}

The DO-controlled fed-batch strategy has the advantage to highlight the environmental oscillations at the level of the dissolved oxygen, but also at the level of the glucose. Fig. 3 shows how the DO profile can be used to extract some informations about the glucose availability. The glucose is fed to the culture when the DO level is above the set-point of $30 \%$. In our case, the glucose is considered to be available when the set point is reached and during the whole DO drop. At this time, the glucose is considered to be limiting or depleted as the DO is rising and until the set-point is reached. This local minimum represents thus the transition between a glucose consumption sequence and a glucose depletion sequence. A custom MatLab (Matworks) code has been written in order to analyze a given DO profile and to extract informations about the time length of the different environmental sequences: the glucose feed sequences correspond to the successive parts of the DO curve above the $30 \%$ (set point for the fed-batch regulation), the glucose consumption sequences begin at the same time than the corresponding feeding sequence and end when the DO is rising after the initial drop induced by the aerobic glucose metabolism. The glucose consumption rate is calculated for each phases by multiplying the feed time by the feed pump flow rate and the glucose concentration in the feed solution. The amount of glucose obtained is then divided by 


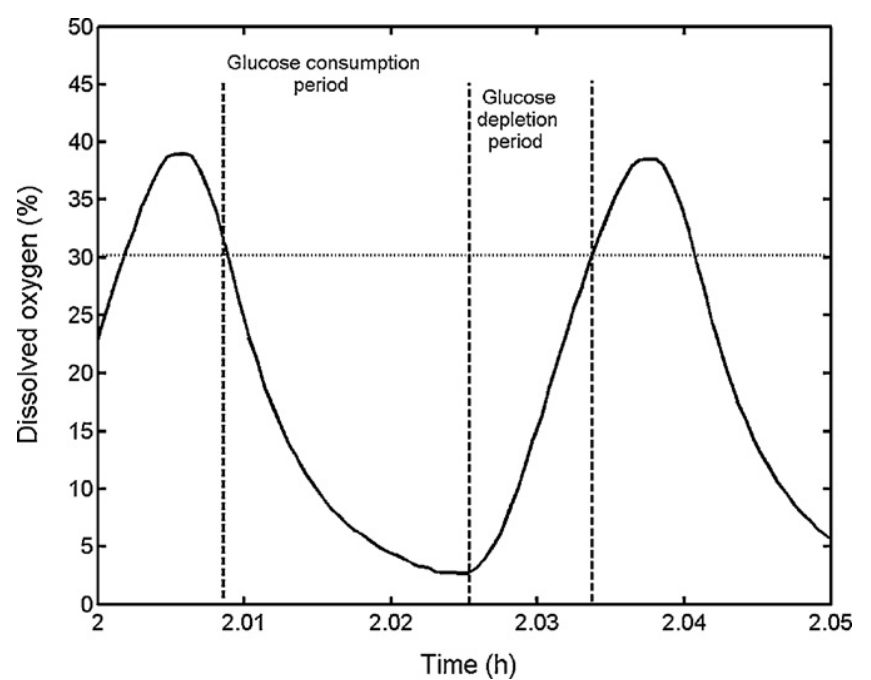

Fig. 3. Typical DO oscillation encountered when glucose is fed intermittently to the culture. The analysis of such profile allows the extraction of informations about the glucose availability in the broth.

the consumption time length of the considered sequence and leads to the glucose consumption rate (in $\mathrm{g} / \mathrm{s}$ ).

Incorporation of the bioreactor hydrodynamics environmental sequences: Markov chain modeling

The Markov chain framework is used frequently to model sequences of stochastic events. The Markov model comprises several states, the transition between these states being governed by probabilities. The Markov framework will be used in our case to model the glucose dynamics and the microbial cells displacement inside a perfectly mixed and a scale-down reactor. In the case of the assumed perfectly mixed reactor (i.e., a 201 stirred reactor without the scale-down apparatus), the situation is very simple. The state space is indeed constituted by a state representing the stirred vessel, and an absorbing state representing the biomass consuming the available substrate (Fig. 4). The state vector can thus be written:

$S=\left[\begin{array}{l}S_{1} \\ S_{2}\end{array}\right]$
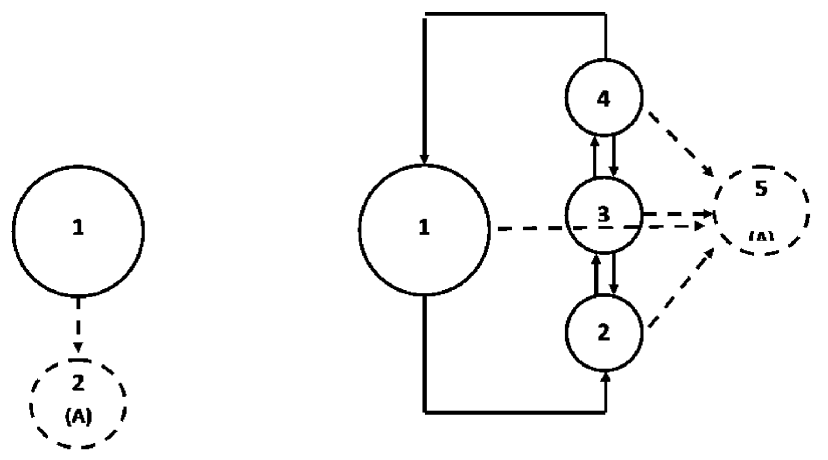

Perfectly mixed reactor

Scale-down reactor

Fig. 4. Schematic representation of the state space of a Markov chain in the case of a perfectly mixed reactor (left) and a scale-down reactor (right). For each case, the state 1 corresponds to a perfectly mixed zone and the state A corresponds to an absorbing state representing the substrate consumption by the microbial population. In the case of the scale-down reactor (right), the states 2, 3 and 4 correspond to the successive flow regions inside the nonmixed part.
$S_{1}$ corresponding to the amount of glucose presents in the volume of the reactor and $S_{2}$ being an absorbing state representing the glucose consumption by the respiring bacteria. The transition matrix collecting all the possible transition probabilities between the different states is written:

$T=\left[\begin{array}{ll}P_{11} & P_{12} \\ P_{21} & P_{22}\end{array}\right]$

with $P_{12}$ being equal to the glucose consumption rate $r_{\text {glu }}$, $P_{11}=1-r_{\text {glu }}$. The absorbing property of the state 2 imposes $P_{21}=0$ and $P_{22}=1$. The situation is more complicated in the case of a heterogeneous bioreactor, i.e. a scale-down reactor (Fig. 4). In this case, the state space comprises 5 states: a state for the mixed part of the reactor; three states in series for the nonmixed part of the SDR and an absorbing state representing the glucose consumption by the microbial population.

$S=\left[\begin{array}{l}S_{1} \\ S_{2} \\ S_{3} \\ S_{4} \\ S_{5}\end{array}\right]$

with $S_{1}$ being the state space corresponding to the mixed part of the reactor, $S_{2}, S_{3}$ and $S_{4}$ being the states corresponding to the nonmixed part and $S_{5}$ being an absorbing state. The transition matrix in the case of the SDR is written:

$$
T=\left[\begin{array}{lllll}
P_{11} & P_{12} & P_{13} & P_{14} & P_{15} \\
P_{21} & P_{22} & P_{23} & P_{23} & P_{25} \\
P_{31} & P_{32} & P_{33} & P_{34} & P_{35} \\
P_{41} & P_{42} & P_{43} & P_{44} & P_{45} \\
P_{51} & P_{52} & P_{53} & P_{54} & P_{55}
\end{array}\right]
$$

By eliminating the transitions that are not physically allowed by the bioreactor hydrodynamics and by considering the absorbing property of the state 5 , the matrix $T$ becomes:

$$
T=\left[\begin{array}{lllll}
P_{11} & P_{12} & 0 & 0 & P_{15} \\
0 & P_{22} & P_{23} & 0 & P_{25} \\
0 & P_{32} & P_{33} & P_{34} & P_{35} \\
P_{41} & 0 & P_{43} & P_{44} & P_{45} \\
0 & 0 & 0 & 0 & 1
\end{array}\right]
$$

The transition probabilities $P_{12}$ and $P_{41}$ are equal respectively to $Q_{\text {pump }} / V_{\text {mixed }}$ and $Q_{\text {pump }} / V_{\text {nonmixed }}$, with $Q_{\text {pump }}$ being the feed pump flow rate, $V_{\text {mixed }}$ and $V_{\text {nonmixed }}$ being respectively the volume of the mixed and the nonmixed part of the SDR (these value have been previously validated by residence time distribution experiments). The backmixing probabilities, $P_{43}$ and $P_{42}$, have been extracted from mixing time experiments performed on the nonmixed part of the SDR by planar laser induced fluorescence. The probabilities $P_{15}, P_{25}$, $P_{35}$ and $P_{45}$ of being absorbed by state 5 are equal to the glucose consumption rate $r_{\text {glu }}$. The remaining probabilities correspond for a given state $i$ to remain in this state $P_{i i}$. This kind of transition probability is calculated in order to give the sums of the rows of the matrix $T$ equal to one (balancing condition for the transition matrix):

$P_{i i}=1-\Sigma_{j} P_{i j}$ 


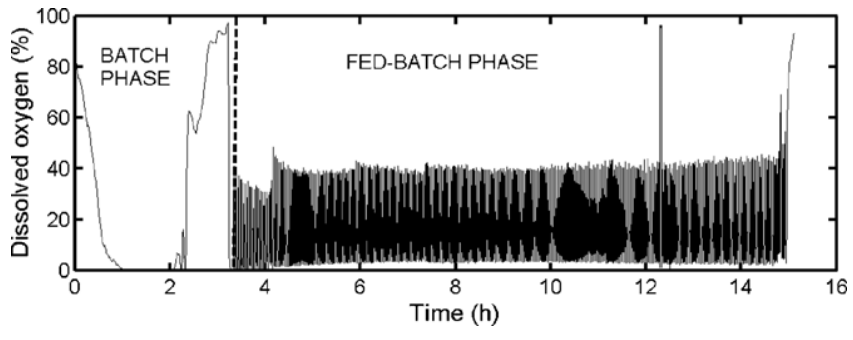

Fig. 5. Dissolved oxygen profile during the culture of $S$. cerevisiae in a 201 stirred bioreactor considered as perfectly mixed.

The complete Markov chain procedure involves the computation of the state space vector $S$ by its successive multiplication with the transition matrix $T$ on discrete time increments $\Delta t$ (here chosen as equal to $1 \mathrm{~s})$ :

$S_{t+\Delta t}=T \cdot S_{t}$

In order to get a realistic picture of the bioreactor glucose dynamics, the complete equation is:

$S_{t+\Delta t}=T \cdot S_{t} \cdot V+S_{a, t}$

With $V$ being a vector comprising the volume of each state and $S_{a}$ being the vector representing the feed addition of glucose with time. The variation of $S_{a}$ with time is governed by the glucose feed sequences calculated previously. In the case of the SDR reactor, microbial cells are allowed to circulate between several flow regions (represented here by the states $S_{1}$ to $S_{4}$ ) and it is thus important to take this characteristic into account when observing the environmental transitions experienced by the cells. The position of a given microbial cell in the SDR can take 4 distinct values corresponding to the state of the Markov chain $S_{1}, S_{2}, S_{3}$ or $S_{4}$ according to the corresponding flow region visited. The state $S_{5}$ is not considered here given the fact that, unlike the glucose, the microbial
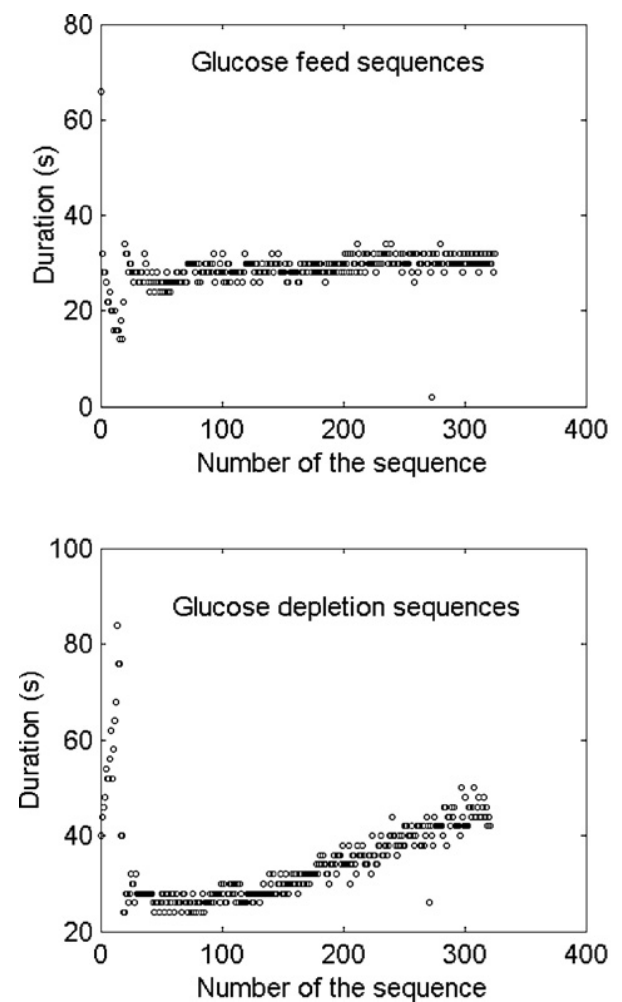

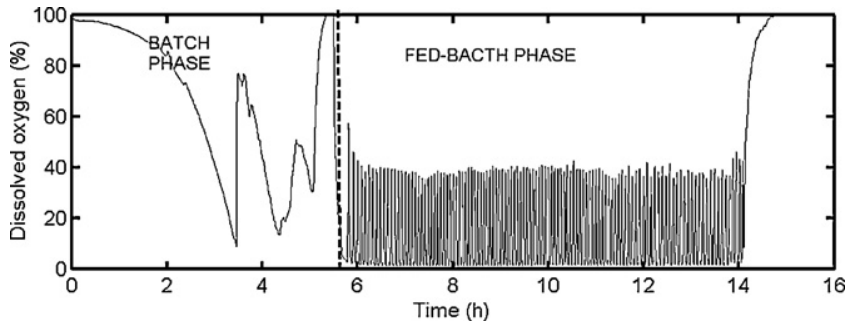

Fig. 7. Dissolved oxygen profile during the culture of S. cerevisiae in a SDR.

cell are not absorbed or consumed during the process. The circulation of a microorganism inside the reactor can thus be simulated by considering the following transition matrix:

$T=\left[\begin{array}{llll}P_{11} & P_{12} & 0 & 0 \\ 0 & P_{22} & P_{23} & 0 \\ 0 & P_{32} & P_{33} & P_{34} \\ P_{41} & 0 & P_{43} & P_{44}\end{array}\right]$

The simulation of the successive position of a microorganism does not follow the iterative process depicted by Eqs. (7) and (8), but a Monte Carlo procedure based on the distribution given by the transition matrix (Eq. (9)). In the transition matrix, each row represents the probability distribution for a microorganism to reach the next state or flow region (e.g., the first row of the matrix depicted by Eq. (9) tells that the microorganism presents in the first state can stay in this state with a probability $P_{11}$ and can reach the second state with a probability $P_{12}$. It is impossible to reach directly the state 3 and 4 from the first state given that the respective probabilities are null).
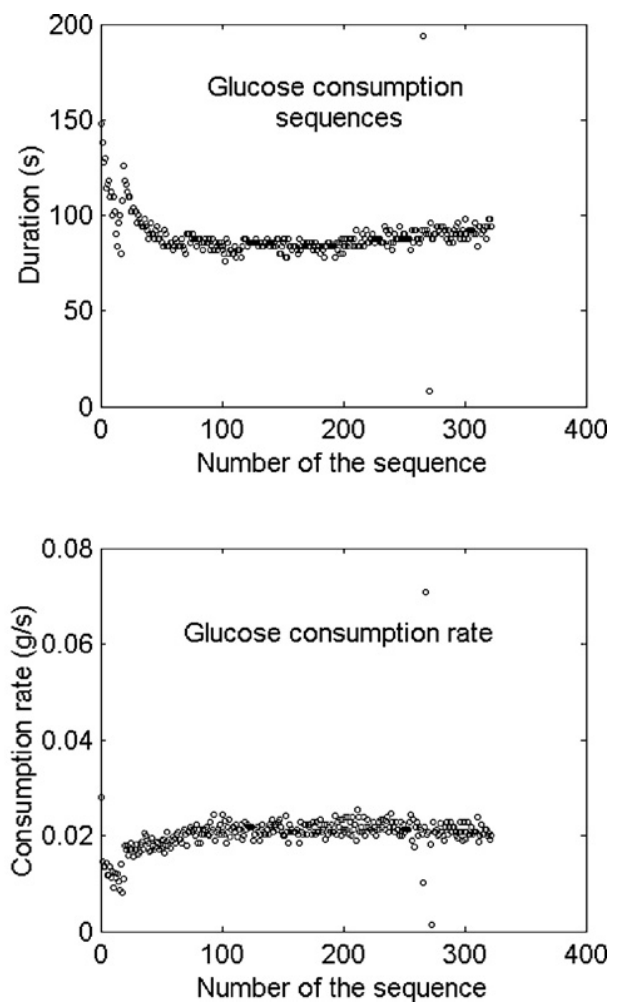

Fig. 6. Evolution of the time lengths of the environmental sequences and the glucose consumption rate in a well-mixed reactor (the numbers of the sequences are presented according to the chronological appearance during the culture). 

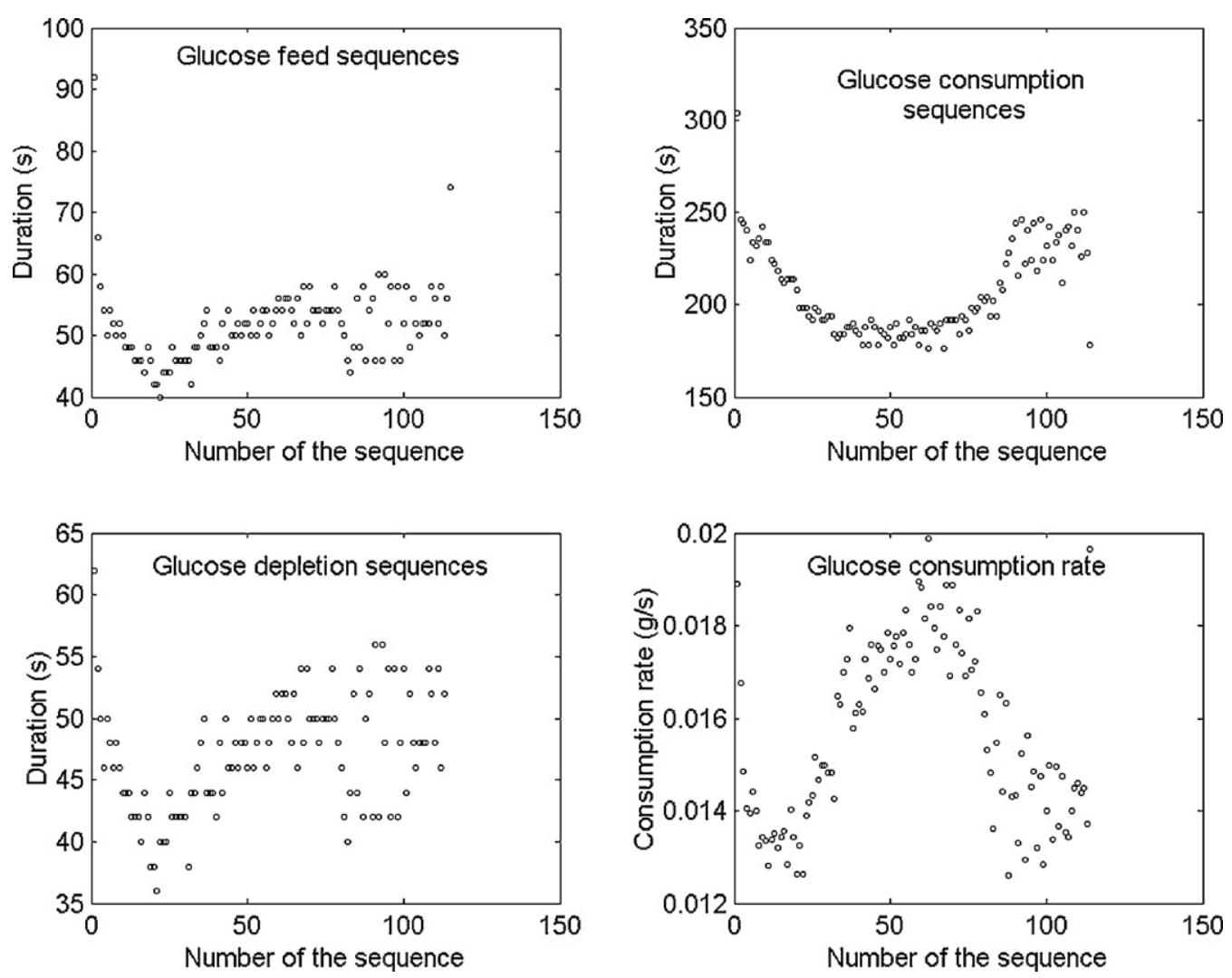

Fig. 8. Evolution of the time lengths of the environmental sequences and the glucose consumption rate in a well-mixed reactor (the numbers of the sequences are presented according to the chronological appearance during the culture).

\section{Results and discussion}

Analysis of the environmental sequences in the case of a well-mixed bioreactor

In a first time, it will be considered that the bioreactor is perfectly mixed, i.e. the glucose added at time $t$ is instantaneously homogenized in the whole volume of the reactor and there is no dissolved oxygen gradient inside the reactor. Fig. 5 shows the DOprofile of a culture performed in a 201 stirred bioreactor. For this reactor which is well mixed (mixing time of about $3 \mathrm{~s}$ ), the above mentioned hypothesis can be applied. It will be shown that, by considering this assumption and by using an appropriate computational method for the analysis of the dissolved oxygen profile, it is relatively easy to reconstruct the glucose profile. The DO fluctuations occurring during the fed-batch phase is processed in order to extract information about the environmental transitions, i.e. in our case depending on the glucose and the dissolved oxygen availability, experienced by the microbial cells during the culture (see Materials and methods). The data related to the environmental sequences are presented in Fig. 6 which shows that the glucose consumption sequences and the glucose depletion sequences increase with time during the course of the culture (from 80 to $90 \mathrm{~s}$ in the case of the consumption sequences and from 30 to $50 \mathrm{~s}$ in the case of the depletion sequences) as the substrate demand increases with the size of the microbial population. The calculated glucose consumption rate over the different environmental sequences is constant (Fig. 6). These results suggests that the microbial cells are submitted to alternative sequences of glucose availability and depletion (feast and famine) imposed by the oxygen transfer capacity of the system. This capacity being limited to a maximal value that has been reached during the experiment, the microbial growth increases linearly with time, as suggested by the constant amount of glucose added during the culture (Fig. 6). The results suggest that, in the case of a well-mixed reactor, the microbial reactions are relatively constants according to the regularity of the time length of the different environmental sequences (Fig. 6). In the following section, the impacts of hydrodynamic imperfections on the process will be investigated.

\section{Analysis of the environmental sequences in a non-perfectly mixed bioreactor: scale-down approach}

The same kind of fed-batch experiment has been performed in a heterogeneous mixed bioreactor by using a scale-down strategy. In the SDR, the glucose feed solution is added at the level of the inlet of the nonmixed part of the reactor, inducing the accumulation of glucose in this zone and a delay at the level of the DO-control strategy. The DO profile and the analysis of the environmental sequences in this reactor are shown respectively in Figs. 7 and 8. The first observation made when comparing Fig. 4 and Fig. 6 is the drop at the level of the number of feed phases when operating in the SDR. This effect can be attributed to the nonmixed part in which glucose is accumulated. This phenomenon occurs without any response at the level of the oxygen probe located in the mixed part of the SDR and results in an increase of the time length of the feeding period. A second important observation is that the time lengths of the different environmental sequences vary greatly during the culture. It is thus important to incorporate these fluctuations induced by the physiological reactions at the level of the hydrodynamic models. This kind of limitation exerted by the lack of knowledge at the level of the microbial reactions in fluctuating environment has been previously reported in another work combining the bioreactor hydrodynamic to the microbial physiology (Lapin et al., 2004; Schmalzriedt et al., 2003). This problem is presently overcome by incorporating directly the reaction sequences at the level of the 

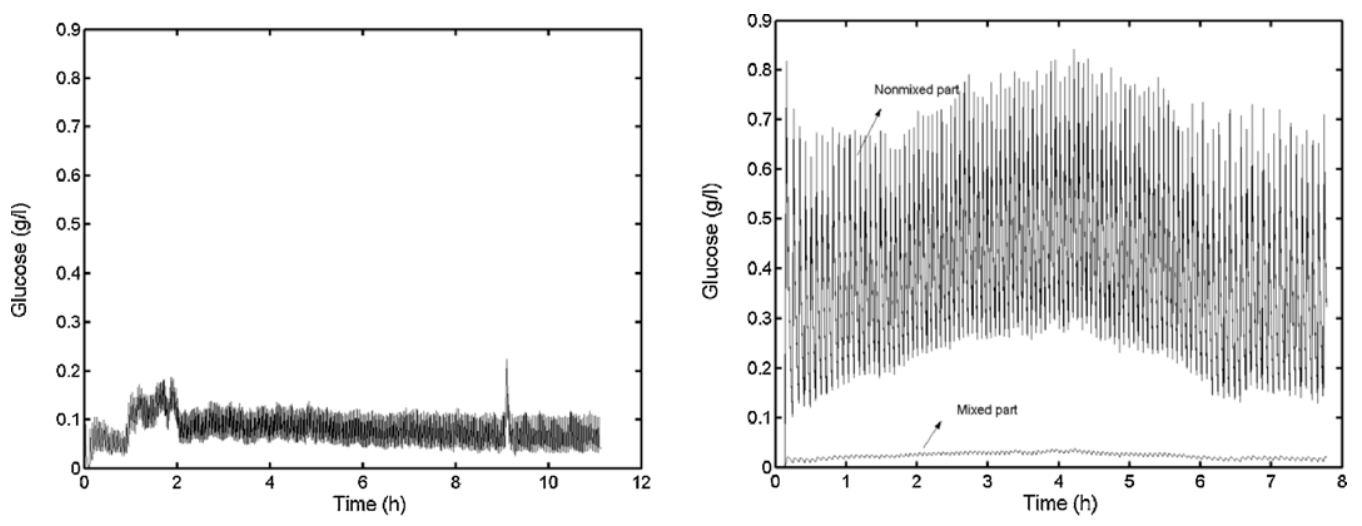

Fig. 9. Reconstructed glucose profile in the well-mixed bioreactor (left) and in the SDR (right).

hydrodynamic model, as it will be shown in the next section. In the previous section, considering the perfectly mixed hypothesis, it has been assumed that the microbial cells encounter exactly the same periodic oscillations in glucose and dissolved oxygen. However, in a scale-down reactor and especially when operating in fed-batch mode, substrate gradients are likely to appears. This hydrodynamic phenomena, superimposed to the fact that the microbial cells circulate in the different fluid zones of the reactors lead to the appearance of stochastic behavior at the level of the substrate profiles experienced by the microbial cells. These phenomena will be studied in details in the following section.

\section{Markov chain formulation of the environmental transitions occurring in well-mixed reactor and in SDR}

In the case of the culture performed in the classical 20 litters bioreactor, the perfectly mixed hypothesis has been adopted. The corresponding hydrodynamic Markov chain is shown in figure with the transition matrix at equation. In the case of the SDR, the Markov chain hydrodynamic model (Fig. 4) is a little more complex with a transition matrix given by Eq. (5). The Markov chain algorithm has been used for each reactor and the resulting glucose profiles are shown in Fig. 7. This figure shows two fundamentally different environmental conditions. In the case of the well-mixed reactor, the glucose level oscillates round a value of $0.1 \mathrm{~g} \mathrm{l}^{-1}$, this value decreasing as the sugar demand increases with time. In the case of the scale-down experiment, two distinct situations are encountered in function of the localization inside the reactor. In the mixed part, the glucose level slightly oscillates around a very low value, whereas in the nonmixed part the glucose levels are significantly higher and exhibit marked oscillations. In the case of the well-mixed reactor, the microbial cells are assumed to experience the same environmental conditions (Fig. 9, left). This hypothesis is valid because the mixing time of the system is very low and no significant gradients occur in the reactor. This is not the case of the SDR for which the cells can be located at the level of the mixed or the nonmixed part in function of time. In this case, the displacement of the cells from a part of the SDR to another has to be considered in order to calculate the experienced concentration profiles. This has been done by performing a Monte Carlo Markov chain simulation on the basis of the transition matrix depicted by Eq. (9). The Monte Carlo procedure allows the computation of the position of a given cell in function of time and the concentration profile is obtained by superimposing the results to the concentration gradient calculated in Fig. 9 (right). Fig. 10 been obtained by cumulating the time lengths passed by a given microbial cell in a state of glucose excess or depletion (a threshold of $0.02 \mathrm{~g} \mathrm{l}^{-1}$ has been considered for the glucose depletion and a threshold of $0.1 \mathrm{~g} \mathrm{l}^{-1}$ has been considered for the glucose excess possibly leading to an overflow metabolism).
For the SDR simulation, 1000 cells have been numerically tracked over the course of the culture. Fig. 10 shows clearly that the microbial cells are subjected to more severe period of glucose starvation in the case of the SDR than in the well mixed one. The simulations can be thus used to calculate a better feed profile for the SDR. This can be achieved for example by increasing the length of the feed period. In this case, care should be taken in order to limit the occurrence of an overflow metabolism in the nonmixed part of the reactor. On a more fundamental basis, an important question has to be addressed: how the stochastic behavior at the level of the environmental conditions experienced by the microbial cells can be translated at the physiological level by the appearance of distinct populations exhibiting their own phenotype as reported in several work involving a scale-down strategy (Boswell et al., 2003; Neubauer and Junne, 2010; Sweere et al., 1988). These complex physiological reactions involves sensing mechanisms and regulatory network (including feedback loops and toggle switch motifs) which can be at the basis of the development of distinct phenotypes inside the microbial populations. However, the physiological capacity (and notably, the protein content) of the individual microbial cells plays an important role in the process. At this level, we have not enough informations to predict the impact of the environmental fluctuations on the microbial population, but the numerical procedures presented in this work will be extended in the future to the environmental sensing phenomena at the level of theindividual

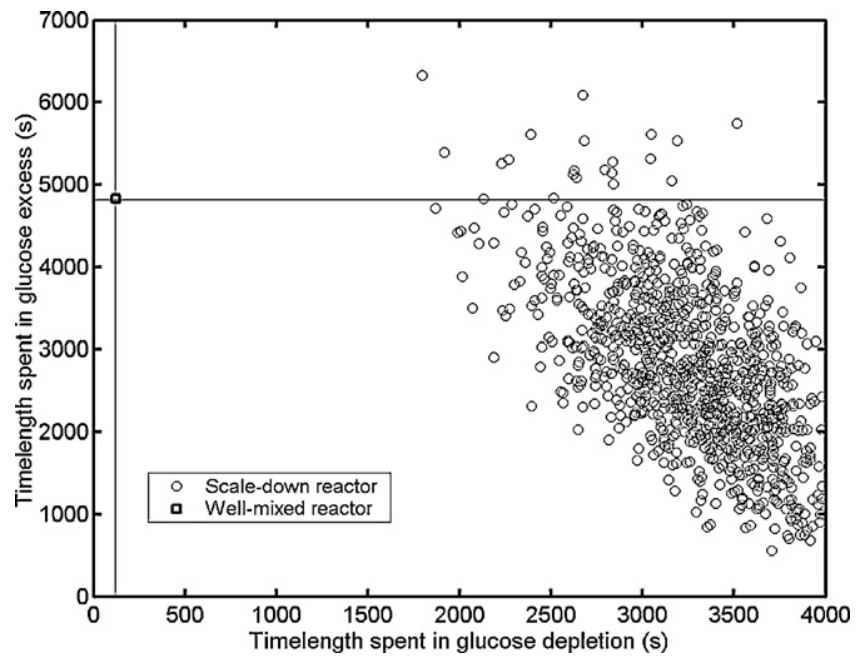

Fig. 10. Comparison of the cumulated glucose depletion and excess time lengths in the SDR (circles) and the well-mixed reactor (square). The well-mixed case is represented by a single point (square) since the environmental conditions are assumed to be perfectly homogenous and the microbial cells encounter exactly the same conditions. 

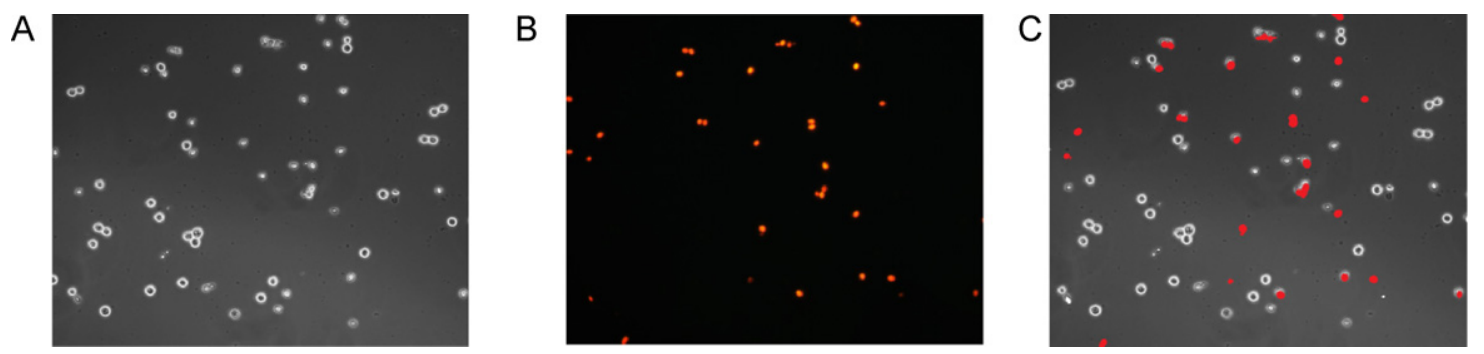

Fig. 11. S. cerevisiae cells stained with propidium iodide (PI): (A) classical microscopy; (B) fluorescence field with cells stained with PI appearing in red; (C) image (A) and (B) merged. PI diffuses across damaged membrane and allows the detection of non viable cells in the population. (For interpretation of the references to color in this figure legend, the reader is referred to the web version of the article.)

microbial cells and to the microbial population segregation mechanisms.

Perspective: flow cytometry as a complimentary tool to investigate yeast physiology at the single cell level

The methodology presented previously is very interesting in order to characterize the extracellular conditions perceived by the microbial cells in bioreactors. The modeling framework presented follows a very simple mathematical formalism allowing to consider the coupling of this actual hydrodynamic model with a biological model representing the physiological response of the yeast cells. However, this response is actually not known since it involves several characteristic times (from the synthesis of mRNA molecules to the synthesis of proteins with the resulting physiological consequences). Our simulations have shown clearly that the microbia cells can be exposed to very different extracellular conditions and these stochastic events make the picture much more complicated. Indeed, when considering a heterogeneous reactor (such as those encountered at the industrial scale), the microbial population has to be considered as segregated. Several techniques can be used in order to investigate the heterogeneities of the microbial population exposed to process related stress. The most straightforward way to perform such analysis relies on the use of fluorescence microscopy. Stained cells can be easily visualized by this way (Fig. 11), but this method is time consuming and cannot be applied in order to study large amounts of microbial cells. An emerging method for the rapid quantification of cytological properties among a microbial population requires the use flow cytometry (Diaz et al., 2010). This method is based on microfluidic devices in order to isolate cells in a vein of fluid with a high throughput (several thousands of cells can be analyzed per seconds). By this way, cell can be individually analyzed by optical devices. Several parameters can be analyzed for a single cell. The first category includes the direct parameters, i.e. those not requiring a preliminary staining of the cells, i.e. FSC (forward scatter proportional to the cell size) and SSC (side scatter indicatives of the granularity of the cell). The second category includes the indirect parameters, i.e. those requiring a staining step, i.e. FL1, FL2 and FL3 corresponding to the green, yellow and red fluorescence emitted by the stained cells (more sophisticated flow cytometers include

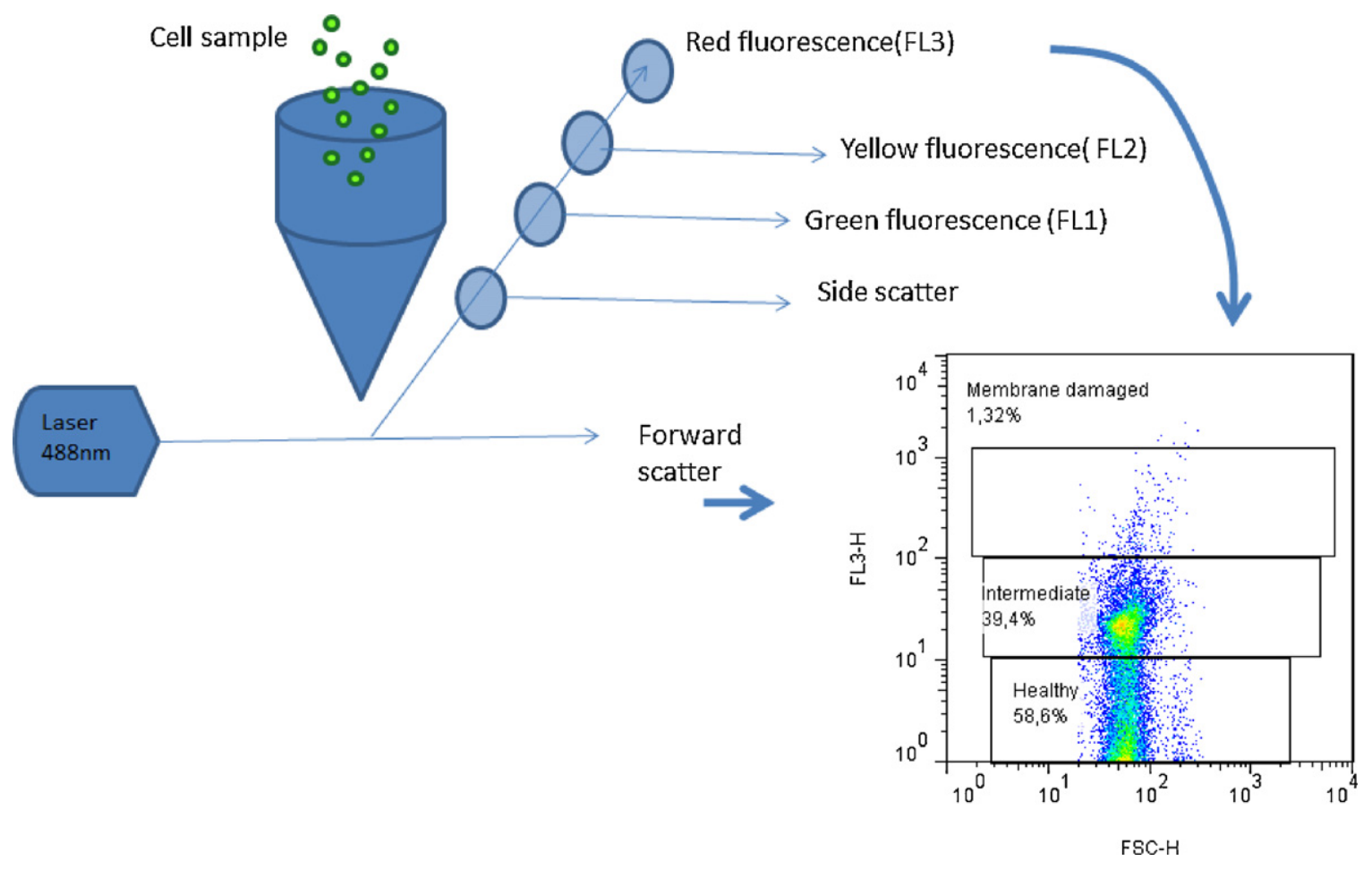

Fig. 12. Principle of flow cytometry. Cells are separated and individually excited by a laser (classically running at a wavelength of $480 \mathrm{~nm}$, but other wavelength can be considered). The flow cytogram shows a dotplot of a yeast population corresponding to the same sample considered before for the microscopic study. Each dot corresponds to a single yeast cells with different FSC (size) and FL3 (red fluorescence) parameters. Dotplot analysis shows that the population is heterogeneous at the level of the FL3 parameter with a healthy (non stained with PI) an intermediate (moderately stained with PI) and damaged (fully stained with PI) subpopulations. (For interpretation of the references to color in this figure legend, the reader is referred to the web version of the article.) 
more fluorescence channels). Since several parameters can be analyzed simultaneously for each single cells passing through the flow cytometer, a statistically relevant description of the population can be obtained by this way (Fig. 12).

\section{Conclusion}

It has been shown that the DO-fluctuations induced by the fedbatch regulation can be exploited to give a better insight at the level of the environmental transitions experienced by the microbial cells during the process. The time lengths of the environmental sequences are significantly different for a well-mixed and scaleddown reactor. These time lengths govern the frequency at which the microbial cells are exposed to the glucose availability and depletion sequence. This frequency is lower for the SDR, suggesting that the starvation effect is more important for this kind of reactor. In addition, the microbial cells in a scale-down reactor can be submitted to stochastic environmental events the occurrence of glucose availability and depletion sequences. A Monte Carlo procedure superimposed to the classical Markov chain development has allowed us to simulate the glucose concentration profiles experienced by the microbial cells in the different parts of the SDR. The results obtained have confirmed the fact that the individual microbial cells are subjected to severe glucose limitation in the SDR under a DO-controlled fed-batch strategy. The Markov chain modeling procedure presented in this work exhibits several advantages. First, the simple discrete mathematical formalism which makes it very easy to implement in any software package (MatLab 7.1. has been used in this work). Second the capacity of the model to capture the stochastic environmental events occurring during a culture. Third, the model can be run in order to perform single cell simulation. Concerning this last point, it will be interesting to use the Markov chain model to make the link between the bioreactor hydrodynamics and the segregation at the level of the microbial population. Indeed, the stochastic external fluctuations can be an important factor leading to the appearance of sub-populations exhibiting distinct physiological states. Several works have shown segregation appearance at the level of the population of $S$. cerevisiae cultivated in heterogeneous conditions. This segregation is mainly induced by the appearance of non-dividing but still metabolically active cells (also called viable but non culturable or VBNC) which have a significant impact on the course of the bioprocess. These subpopulations can be easily analyzed by flow cytometry.

\section{References}

Boswell, C.D., Nienow, A.W., Gill, N.K., Kocharunchitt, S., Hewitt, C.J., 2003. The impact of fluid mechanical stress on Saccharomyces cerevisiae cells during continuous cultivation in an agitated, aerated bioreactor: its implication for mixing $i$ the brewing process and aerobic fermentation. Transactions of the Institution of Chemical Engineers, Part C: Food and Bioproducts Processing 81, 23-32.

Cannizzaro, C.V.S., von Stockar, U., 2004. Control of yeast fed-batch process trough regulation of extracellular concentration. Bioprocess and Biosystems Engineering 26, 377-383.

Delvigne, F., Lejeune, A., Destain, J., Thonart, P., 2006. Stochastic models to study the impact of mixing on a fed-batch culture of Saccharomyces cerevisiae. Biotechnology Progress 22, 259-269.

Diaz, M., Herrero, M., Garcia, L.A., Quiros, C., 2010. Application of flow cytometry to industrial microbial bioprocesses. Biochemical Engineering Journal 48, 385-407.

Gasch, A.P., Spellman, P.T., Kao, C.M., Carmel-Harel, O., Eisen, M.B., Storz, G., Botstein, D., Brown, P.O., 2000. Genomic expression programs in the response of yeast cells to environmental changes. Molecular Biology of the Cell 11, 4241-4257.

Geladé, R., Van de Velde, S., Van Dijck, P., Thevelein, J.M., 2003. Multi-level response of the yeast genome to glucose. Genome Biology 4 (11), 233.

Henson, M.A., Müller, D., Reuss, M., 2002. Cell population modelling of yeast glycolytic oscillations. Biochemical Journal 368, 433-446.

Junker, B., 2004. Scale-up methodologies for Escherichia coli and yeast fermentation processes. Journal of Bioscience and Bioengineering 97 (6), 347-364.

Lapin, A., Müller, D., Reuss, M., 2004. Dynamic behavior of microbial populations in stirred bioreactors simulated with Euler-Lagrange methods: traveling along the lifelines of single cells. Industrial and Engineering Chemistry Research 43, 4647-4656.

Lara, A.R., Galindo, E., Ramirez, O.T., Palomares, L.A., 2006. Living with heterogeneities in bioreactors - understanding the effects of environmental gradients on cells. Molecular Biotechnology 34, 355-381.

Müller, S., Harms, H., Bley, T., 2010. Origin and analysis of microbial population heterogeneity in bioprocesses. Current Opinion in Biotechnology 21, 100-113.

Müller, S., Lösche, A., 2004. Population profiles of a commercial yeast strain in the course of brewing. Journal of Food Engineering 63, 375-381.

Neubauer, P., Junne, S., 2010. Scale-down simulators for metabolic analysis of largescale bioprocesses. Current Opinion in Biotechnology 21, 114-121.

Patnaik, P.R., 2006. External, extrinsic and intrinsic noise in cellular systems: analogies and implications for protein synthesis. Biotechnology and Molecular Biology Review 1 (4), 121-127.

Schmalzriedt, S., Jenne, M., Mauch, K., Reuss, M., 2003. Integration of physiology and fluid dynamics. Advances in Biochemical Engineering 80, 19-68.

Sweere, A.PJ.MJ.R, Janse, L, Luyben, K.C.A.M., Kossen, N.W.F., 1988. Experimental simulation of oxygen profiles and their influence on Baker's yeast production. Part I. One-fermentor system. Biotechnology and Bioengineering 31, 567-578. 